\title{
CARACTERIZACIÓN DE LA DIVERSIDAD DE MOLUSCOS BENTÓNICOS SUBLITORALES EN ISLA CARLOS III Y ÁREAS ADYACENTES, ESTRECHO DE MAGALLANES, CHILE
}

\author{
DIVERSITY CHARACTERIZATION OF SUBTIDAL BENTHIC MOLLUSCS FROM \\ CARLOS III ISLAND AND ADJACENT AREAS, STRAIT OF MAGELLAN, CHILE
}

\author{
Cristian Aldea ${ }^{1}$, Sebastián Rosenfeld ${ }^{2} \&$ Javiera Cárdenas ${ }^{3}$
}

\section{RESUMEN}

A pesar del conocimiento existente sobre ecología del bentos marino en el estrecho de Magallanes, pocos estudios han sido enfocados a la malacofauna. Durante abril y junio de 2007 se realizaron campañas en una extensiva zona ubicada en la Isla Carlos III y áreas adyacentes, en el marco del proyecto "Diagnóstico del Macrobentos en el Área Marina Costera Protegida de Múltiples Usos Francisco Coloane". Mediante buceo autónomo se recolectaron muestras bentónicas sublitorales (5-20 m de profundidad) en 37 sitios, dentro de cuadrantes de 0,25 $\mathrm{m}^{2}$. La fauna obtenida fue tamizada, fijada y preservada en etanol. Los moluscos fueron separados, contabilizados e identificados a nivel de especie. A partir de matrices de datos se evaluó la riqueza, abundancia y diversidad, y se analizaron sus agrupamientos y ordenamientos en ensambles o grupos, estudiando sus especies más comunes y preferentes, y las que más contribuyeron a la similitud. A partir de los 4613 ejemplares estudiados, se identificaron 101 especies: 11 Polyplacophora, 59 Gastropoda y 31 Bivalvia. Las especies más abundantes fueron el gastrópodo Margarella violacea (560 individuos) y el bivalvo Neolepton yagan (389). Los valores más altos de riqueza y abundancia se registraron en la Península de Brunswick, mientras que los mayores valores de diversidad se presentaron en el Canal Jerónimo e Islas Charles. El agrupamiento de estaciones demostró la presencia de cuatro ensambles significativos con un nivel de similitud superior al 20\%, no determinándose un patrón geográfico de organización. El gastrópodo Xymenopsis muriciformis contribuyó notoriamente a la similitud de los ensambles, mientras que Trophon pallidus estuvo presente en todos éstos y el bivalvo Aulacomya atra fue la especie con mayor presencia (17 estaciones). La alta diversidad registrada y los agrupamientos

1 Centro de Estudios del Cuaternario de Fuego-Patagonia y Antártica (Fundación CEQUA); Universidad de Magallanes; Av. 21 de Mayo 1690, Casilla 737, Punta Arenas, Chile. cristian.aldea@cequa.cl

2 Laboratorio de Macroalgas Antárticas y Subantárticas, Universidad de Magallanes, Avenida Bulnes 01855, Casilla 113-D, Punta Arenas, Chile.

3 Centro de Ciencias Ambientales EULA, Universidad de Concepción, Casilla 160-C, Barrio Universitario s/n, Concepción, Chile. 
obtenidos sugieren considerar parámetros abióticos en futuras investigaciones y una mayor cantidad de muestreos en áreas adyacentes.

Palabras clave: Área Marina Costera Protegida de Múltiples Usos Francisco Coloane, Gastropoda, Bivalvia, Polyplacophora, ecología, bentos, ensambles.

\section{ABSTRACT}

Despite the knowledge of marine benthic ecology in the Strait of Magellan, few studies have been focused on malacofauna. During April and June (2007), an extensive region on Carlos III Island and adjacent areas were studied under the project "Diagnosis of Macrobenthos in the Multiple-use Marine Protected Area Francisco Coloane". Subtidal benthic samples (5-20 m deep) were collected by means of SCUBA diving in 37 sites within quadrants of $0.25 \mathrm{~m}^{2}$. The fauna obtained was sieved, fixed and preserved in ethanol. Molluscs were sorted, quantified and identified to species level. From data matrix the richness, abundance and diversity was evaluated, and analyzed the clustering and scaling in assemblages or groups, studying the common and preferential species, and those that most contribute to the similarity. From the 4,613 individuals studied, 101 species were identified: 11 Polyplacophora, 59 Gastropoda and 31 Bivalvia. The most abundant species were the gastropod Margarella violacea (560 individuals) and the bivalve Neolepton yagan (389). The highest values of richness and abundance were recorded in the Brunswick Peninsula, while the highest values of diversity were presented in the Jerónimo Channel and Charles Islands. The stations clustering showed the presence of four significant assemblages with a similarity level $>20 \%$, not determining it a geographic pattern of organization. The gastropod Xymenopsis muriciformis contributed significantly to the assemblages similarity, while Trophon pallidus was present at all assemblages and the bivalve Aulacomya atra was the most presented species (17 stations). The high diversity and the obtained organization suggest considering abiotic parameters in future researches and a higher number of samples from adjacent areas.

Key words: Marine Protected Area Francisco Coloane, Gastropoda, Bivalvia, Polyplacophora, ecology, benthos, assemblages.

\section{INTRODUCCIÓN}

El estrecho de Magallanes, inserto en un sistema de fiordos y canales, destaca como el canal más importante de la Región de Magallanes, correspondiendo a un corredor bioceánico de $\sim 580 \mathrm{~km}$ influenciado por masas de agua provenientes de los océanos Pacífico, Atlántico y Antártico (Antezana 1999). Entre las características más notables, el estrecho de Magallanes puede ser dividido en tres micro-cuencas: i) Oriental, ubicada entre la entrada del Océano Atlántico y la Segunda Angostura, ii) Central desde Segunda Angostura hasta la Isla Carlos III, y iii) Occidental desde la Isla Carlos III hasta la entrada del Océano Pacífico (Valdenegro \& Silva 2003). La formación de estas micro-cuencas son el resultado de procesos tectónicos y glaciares que generaron un paisaje complejo donde la biota marina es representativa, encontrándose una alta variedad y heterogeneidad de hábitat marino-costeros que sustentan una gran diversidad de algas e invertebrados (Camus 2001).

En los últimos años los estudios sobre organismos marinos en la Región de Magallanes se han incrementado (ver Ríos et al. 2003). Algunas investigaciones como los cruceros de "Victor Hensen" (ver Arntz \& Gorny 1996) y "CIMAR 3 Fiordos" (Thatje \& Mutschke 1999a) han contribuido a caracterizar la diversidad sublitoral. Sin embargo, concretamente en el estrecho de Magallanes los estudios asociados a diversidad o aspectos ecológicos de la fauna bentónica sublitoral no son numerosos (i.e. Mazzocchi \& Ianora 1991, Lorenti \& Mariani 1997, Chen et al. 1999, Gutt et al. 1999, Mutschke \& Ríos 2006, Montiel et al. 2001, 2007, 2011, Ríos et al. 2003, 2010, Cárdenas 2008, Hromic 2009, Thatje \& Brown 
2009), o bien se centran en un ámbito más amplio (e.g. Arntz \& Gorny 1996, Gerdes \& Montiel 1999, Thatje \& Mutschke 1999b, Montiel et al. 2005, Ríos et al. 2005). En este contexto, los antecedentes sobre las comunidades bentónicas en la región de Magallanes indican que el phylum Mollusca constituye uno de los grupos faunísticos más representativos en términos de abundancia, distribución y diversidad de especies (Ríos et al. 2003).

El primer trabajo de moluscos en la región Magallánica pertenece a King \& Broderip (1832) y hasta la fecha existen varias investigaciones que entregan la cronología sobre historia natural y diversidad de los moluscos (Dell 1971, Linse 1997, 1999, 2002, Reid \& Osorio 2000, Aldea \& Rosenfeld 2011, Aldea et al. 2011). Otros trabajos continúan entregando información sobre taxones específicos (e.g. Pastorino 2005a, 2005b, Zelaya \& Ituarte 2004, Zelaya \& Geiger 2007, Zelaya 2010). Desde el punto de vista ecológico, varios trabajos han entregado antecedentes sobre la malacofauna bentónica (e.g. Ríos \& Gerdes 1997, Mutschke et al. 1998, Ríos \& Mutschke 1999, Ríos et al. 2003, 2005, 2007, 2010). No obstante, existe un conocimiento limitado sobre la diversidad y organización de comunidades de moluscos de las zonas sublitorales someras ( 5-30 m) del Estrecho de Magallanes, por lo tanto, abordar estos aspectos son esenciales para futuros análisis biogeográficos y ecológicos (Fernández et al. 2000), definir aspectos relacionados a la conservación (Gray 1997, Lancellotti \& Vásquez 2000) o determinar una eventual pérdida de diversidad para el funcionamiento del ecosistema (Purvis \& Hector 2000).

Teniendo en cuenta el amplio espectro ecológico y etológico que presentan los moluscos, la caracterización de la diversidad y análisis de sus ensambles bentónicos son pertinentes para contribuir a entender la estructura e interacciones dentro de ensambles más complejos en los cuales estos moluscos interactúan con otros organismos bentónicos. Por esta razón, planteándose las interrogantes de cómo varía la diversidad de moluscos sublitorales de aguas someras en un área determinada del estrecho de Magallanes y de qué manera esta variación se ve reflejada en la formación de ensambles o agrupaciones de sitios afines, el presente trabajo tiene como objetivo caracterizar la biodiversidad y formación de agrupaciones de los moluscos sublitorales de aguas someras en la zona de la Isla Carlos III y áreas adyacentes.

\section{MATERIAL Y MÉTODOS}

Área de estudio

El área de estudio comprende una extensiva zona emplazada en el límite entre la micro-cuenca central y occidental del estrecho de Magallanes, en Isla Carlos III y áreas adyacentes, desde los fiordos y canales contiguos entre el Canal Jerónimo y Paso Tortuoso y hasta el este de las islas Charles y Canal Bárbara (Fig. 1). Toda esta zona está circunscrita al "Área Marina Costera Protegida de Múltiples Usos Francisco Coloane", el cual presenta una superficie aproximada de $672 \mathrm{Km}^{2}$.

Su posición geográfica, influenciada por aguas de la Corriente del Cabo de Hornos (curso sur de la Corriente de Deriva de los Vientos del Oeste) desde el océano Pacífico, en menor medida por aguas del océano Atlántico, por importantes cursos de agua dulce como el Río Batchelor, por aguas de escorrentía con gran contribución de nutrientes y por aporte de la fusión de glaciares que llegan directo al mar, además de una geomorfología típica del sistema de fiordos y canales magallánicos y fueguinos, con marcadas angosturas y desniveles batimétricos, e influencia de fuertes vientos y precipitaciones en una parte importante del año, generan en el área una condición climática y oceanográfica singular y un ecosistema heterogéneo, asociado a procesos de alta productividad biológica y a una riqueza y diversidad específica comparativamente alta (Zaixso et al. 2007).

Toma y procesamiento de muestras y datos

En el marco del Proyecto de diagnóstico del macrobentos en el Área Marina Costera Protegida de Múltiples Usos Francisco Coloane (Zaixso et al. 2007), se recolectaron muestras bentónicas sublitorales (5-20 m de profundidad) en 37 sitios de muestreo durante los meses de abril y junio de 2007. La extracción se realizó manualmente con espátulas mediante buceo autónomo SCUBA, utilizando cuadrantes de $0,25 \mathrm{~m}^{2}$ en tres réplicas. La fauna obtenida fue tamizada a $0,5 \mathrm{~mm}$, fijada en formalina al 5\% amortiguada con borato de sodio a saturación. En laboratorio fue separada a nivel taxonómico mayor y preservado en etanol al 70\%.

Los moluscos obtenidos fueron separados a nivel de morfoespecies, contabilizados e identificados 


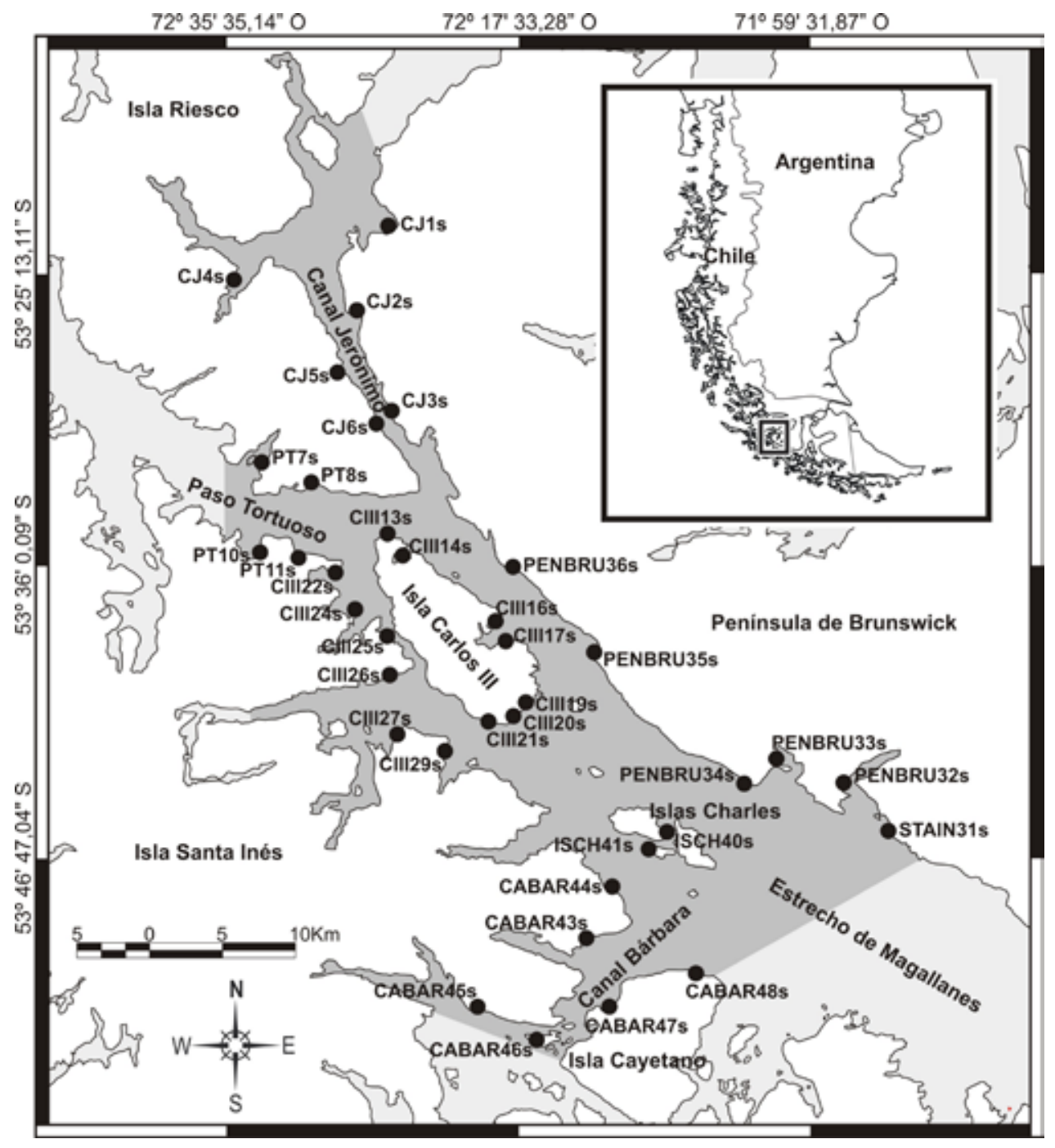

Fig. 1. Situación geográfica del área de estudio en Isla Carlos III y áreas adyacentes, destacando el "Área Marina Costera Protegida de Múltiples Usos Francisco Coloane" (sombra) y señalando los sitios de muestreo donde se obtuvo fauna sublitoral.

al nivel taxonómico más bajo posible (i.e. especies o géneros), utilizando como referencia toda la literatura disponible para los moluscos de la región magallánica y áreas vecinas, incluyendo trabajos de revisiones a taxones específicos (e.g. Zelaya 2004). La información obtenida fue tabulada por estación de muestreo en una base de datos.

Análisis de información

Para cada estación de muestreo fueron calculadas las medidas descriptivas univariantes de abundancia total de individuos $(\mathrm{N})$, número de especies
(S), índice de diversidad de Shannon (H', Shannon 1948) e índice de equidad de Pielou (J', Pielou 1966). Además se calculó la diversidad taxonómica $(\Delta)$ y distinción taxonómica $\left(\Delta^{*}\right)$ según Warwick \& Clarke (1995), incluyendo los niveles taxonómicos de especies, géneros, familias y subclases; previo ajuste taxonómico de los taxones según la base de datos WORMS (Appeltans et al. 2011).

Para determinar los ensambles de moluscos fueron empleadas técnicas multivariantes no paramétricas, utilizando el software PRIMER-E v6.0 (Clarke \& Gorley 2005). Se efectuó la construcción de una matriz de similitud entre los sitios de muestreo 
mediante el coeficiente de similitud de Bray-Curtis (Bray \& Curtis 1957) y luego la clasificación de los sitios mediante el algoritmo de agrupación o dendrograma UPGMA (Rohlf 1963) con agrupación en función de la similitud media de los sitios, a partir de la prueba SIMPROF (Clarke et al. 2008). Se realizó un análisis de ordenación MDS (Kruskal \& Wish 1978). Se determinaron las especies responsables de los agrupamientos de sitios a través del análisis SIMPER (Clarke 1993). Finalmente, se analizaron las diferencias espaciales en la estructura de los ensambles o agrupamientos por medio de la prueba estadística no paramétrica ANOSIM de una vía (Clarke \& Green 1988). Todos los análisis fueron realizados previa transformación de la matriz de datos de número de individuos por estación (abundancias) mediante raíz cuarta (ver Field et al. 1982).
Basados en los agrupamientos o ensambles de los sitios construidos por medio de los análisis multivariantes, se utilizaron los índices de Constancia y Fidelidad (Dajoz 1971) para la clasificación biocenótica de las especies, cuyas fórmulas y niveles fueron reproducidas por Arnaud et al. (2001).

\section{RESULTADOS}

Fueron identificados un total de 4.613 especimenes de moluscos, los cuales pertenecen a tres clases (Gastropoda, Bivalvia y Polyplacophora), 48 familias y 101 especies (Fig. 2). En términos de riqueza de especies, la clase Gastropoda fue la mejor representada (con 59 especies pertenecientes a 26 familias), seguida por la clase Bivalvia (31 especies pertenecientes a 17 familias) y la clase Polyplaco-

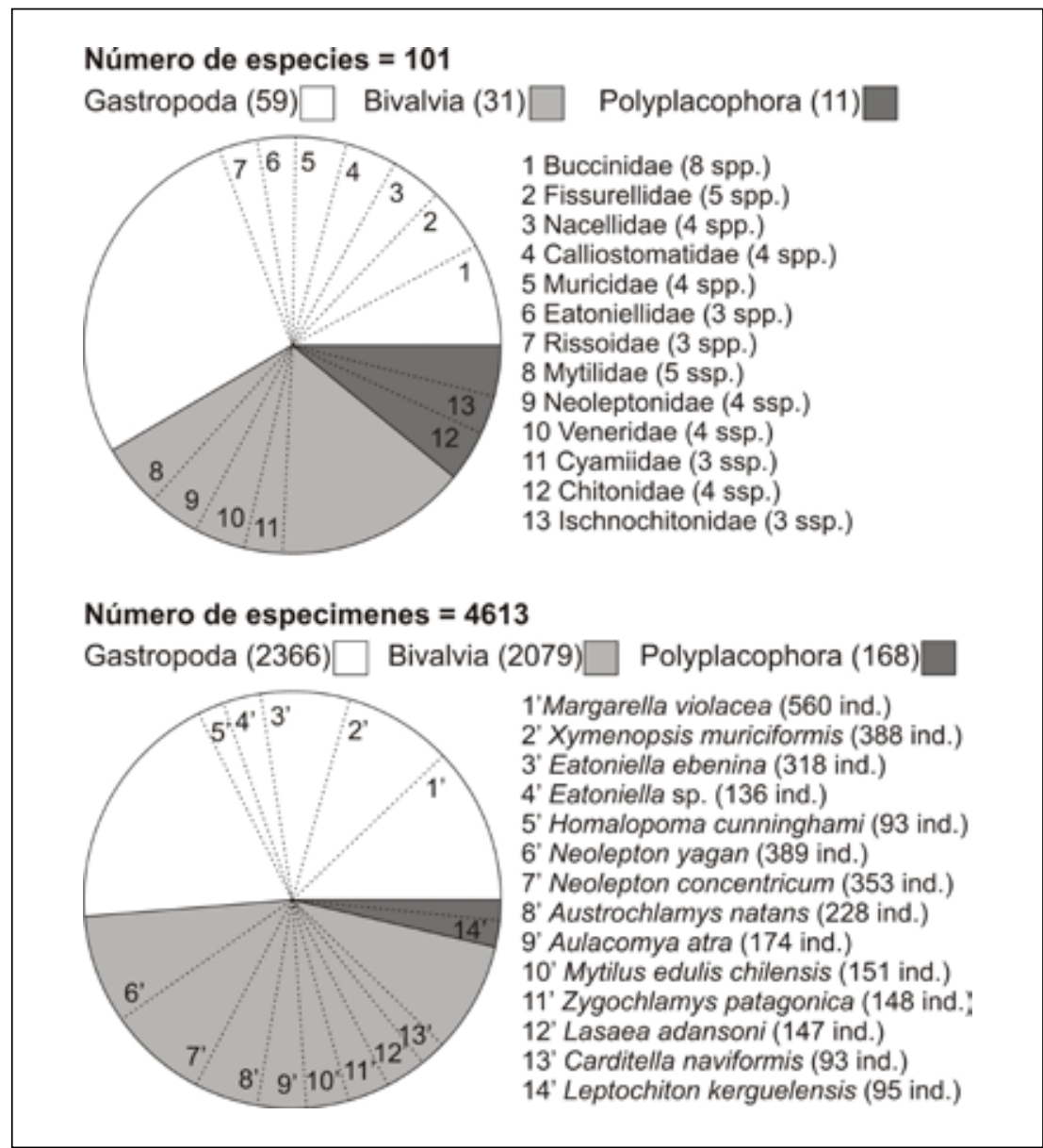

Fig. 2. Número de especies y especimenes de cada clase de moluscos, indicando las familias mejor representadas y las especies más abundantes en Isla Carlos III y áreas adyacentes. 

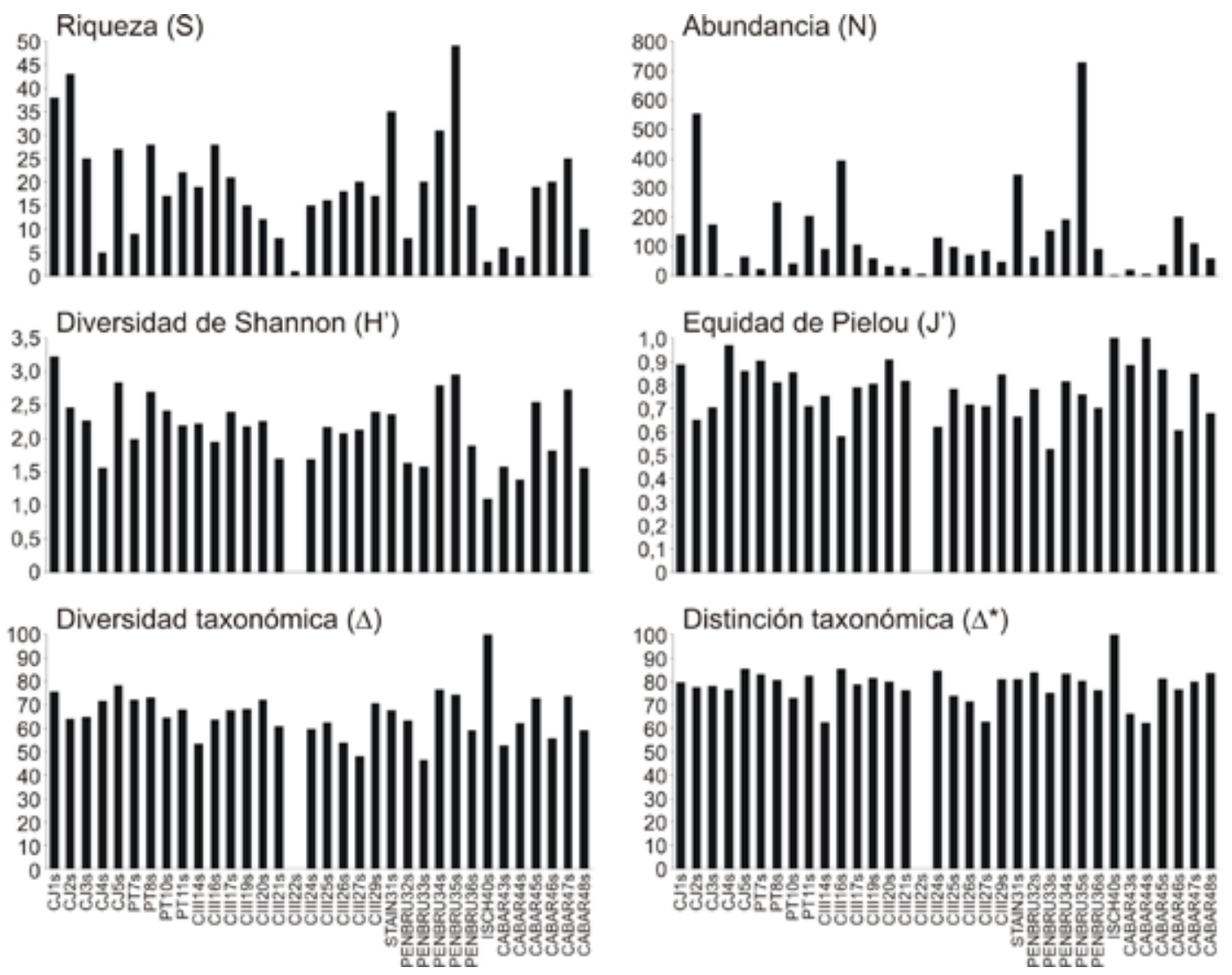

Fig. 3. Parámetros indicadores de diversidad de moluscos en sitios de muestreo en Isla Carlos III y áreas adyacentes.

phora (11 especies pertenecientes 5 familias). La familias más diversas (Fig. 2) fueron los gastrópodos Buccinidae (8 especies), seguidos por Fissurellidae y los bivalvos Mytilidae (5 especies cada una). En términos de abundancia (Fig. 2), la clase Gastropoda acaparó la mayoría de los especimenes (2366) seguido por Bivalvia (2079) y Polyplacophora (168). A nivel de especies, las más abundantes fueron los gastrópodos Margarella violacea (560 individuos), Xymenopsis muriciformis (388) y Eatoniella ebenina (318) y los bivalvos Neolepton yagan (389) y Neolepton concentricum (353), los cuales en conjunto representaron casi el $45 \%$ de todos los individuos del estudio (Fig. 2).

Diversidad

Los valores más altos de riqueza de especies y abundancia fueron registrados en la estación PENBRU35s (49 especies y 729 individuos) en zona de la Península de Brunswick, mientras que la mayor diversidad de Shannon se presentó en la estación CJ1s (valor de 3,223) en el Canal Jerónimo, en tanto que los mayores valores de equidad de Pielou, diversidad taxonómica y distinción taxonómica fueron registrados en la estación ISCH40s (valores de 1,100 y 100, respectivamente) en las Islas Charles (Fig. 3; ver Fig. 1).

No obstante en el Canal Jerónimo también se registraron altos valores de riqueza y abundancia (estación CJ2s) y altos valores de diversidad de Shannon, diversidad taxónómica y distinción taxonómica (estación CJ2s), mientras que en el margen oriental de la Isla Carlos III se registraron altos valores de abundancias y amplitud taxonómica (estación CIII16s; Fig. 3; ver Fig. 1). Los valores más bajos de la mayoría de los parámetros de diversidad se obtuvieron en la estación CIII22s (con sólo 5 individuos de una sola especie) en la zona de la Isla Santa Inés, en la boca del Paso Tortuoso (Fig. 3; ver Fig. 1). 


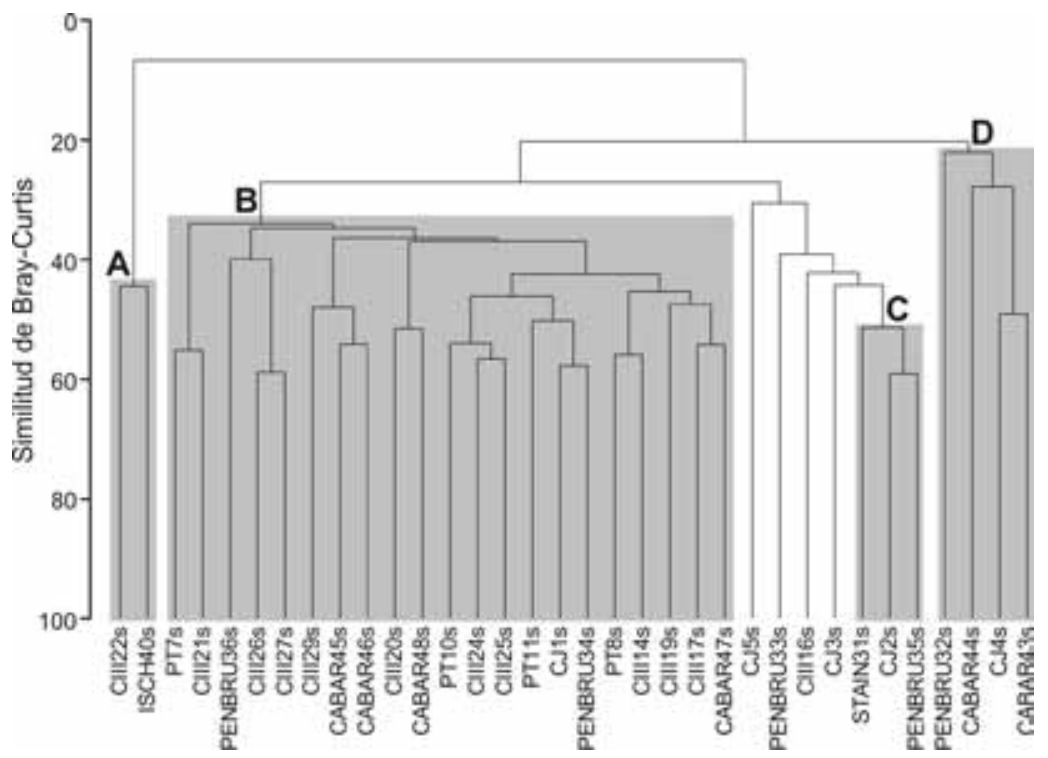

Fig. 4. Ensambles de moluscos en sitios de Isla Carlos III y áreas adyacentes, determinados por el análisis de clasificación basado en el coeficiente de similitud de Bray-Curtis.

Ensambles y estructura de las comunidades

El dendrograma de clasificación -clusterdemostró la presencia de cuatro ensambles de sitios con un nivel de similitud superior al 20\% (Fig. 4): grupo A, compuesto de dos estaciones distantes entre sí (en la zona de la boca del Paso Tortuoso e Islas Charles, ver Fig. 1); grupo B, compuesto de 21 estaciones representadas por todas las zonas del área de estudio, mayormente la Isla Carlos III y la
Isla Santa Inés; grupo C, formado de tres estaciones situadas distantemente entre sí en la Península de Brunswick; y grupo D, formado de cuatro estaciones, tres de ellas en la zona sureste del área de estudio, junto a una de Isla Riesco.

El análisis de ordenación MDS (Fig. 5) exhibió los resultados del dendrograma disponiendo las estaciones agrupadas en sus ensambles, con un coeficiente de estrés de Kruskal de 0,19. Los ensambles fueron ordenados de izquierda a derecha,

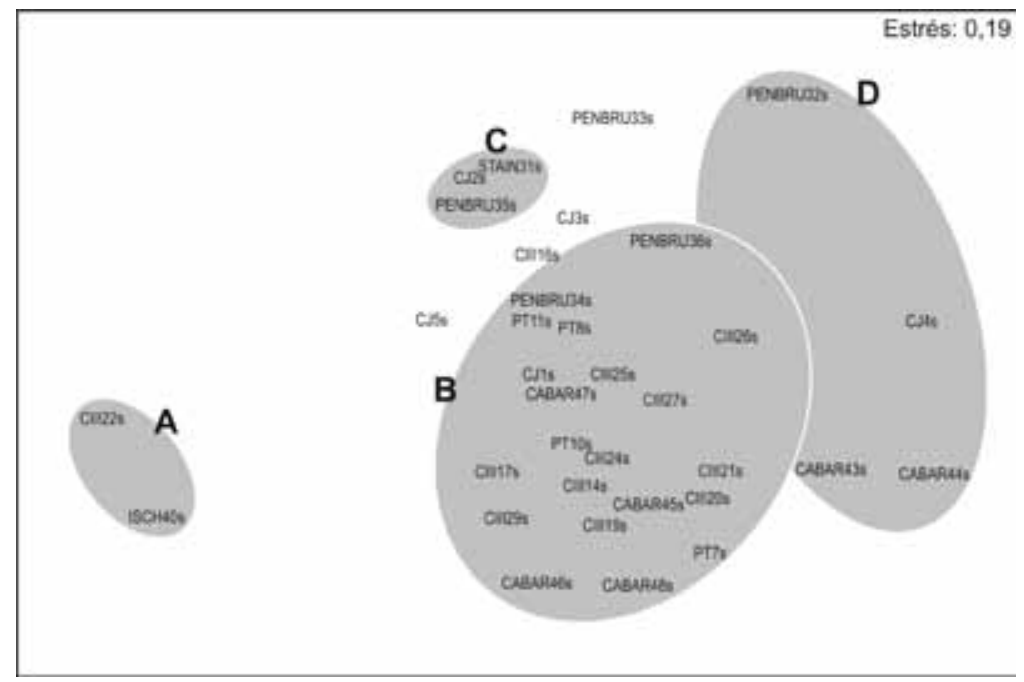

Fig. 5. Ordenación MDS de los ensambles de moluscos en sitios de Isla Carlos III y áreas adyacentes. 
Tabla 1. Prueba estadística ANOSIM comparando los grupos de estaciones ( $\mathrm{R}$ global = 0,807). En negrita se muestra el par de grupos con mayor diferencia significativa.

\begin{tabular}{|c|c|c|}
\hline Grupos & R & Nivel de significación \\
\hline B, C & 0,657 & 0,001 \\
\hline B, D & 0,781 & 0,001 \\
\hline B, A & $\mathbf{0 , 9 9 2}$ & $\mathbf{0 , 0 0 4}$ \\
\hline C, D & 0,852 & 0,057 \\
\hline D, A & 1 & 0,1 \\
\hline
\end{tabular}

pudiéndose identificar el grupo A ubicado a una gran distancia (menor similitud) del resto de los grupos y al grupo D como el grupo menos símil entre sí. El análisis de las similitudes, ANOSIM, arrojó diferencias significativas ( $\mathrm{R}$ global $=0,807 ; p=0,001$ ) en la composición faunística, revelando que existen mayores diferencias significativas entre los pares de grupos B-C, B-D y B-A (Tabla 1).

Los resultados del análisis SIMPER demostraron que el grupo $\mathrm{C}$ es el más similar entre sí, con una similitud promedio de 54\% (Tabla 2). En contraste, el grupo menos similar es el D, con una similitud promedio de 28,56\%. El gastrópodo Xymenopsis muriciformis fue una de las especies que más contribuyó a la similitud de los ensambles (Tabla 2). La disimilitud entre los grupos fue alta (sobre el 73\%) siendo contribuida mayormente por las especies discriminadoras Neolepton yagan y Eatoniella ebenina (para los pares de grupos C vs. A y C vs. D), Aulacomya atra y Margarella violacea (para los pares de grupos B vs. A y B vs. D), y Austrochlamys natans y Xymenopsis muriciformis (para los grupos D vs. A).

Los valores del índice de constancia $(\mathrm{C})$ y fidelidad $(\mathrm{F})$ definieron las especies más y menos comunes en el área de estudio, y las más y menos preferentes para cada grupo de estaciones, respectivamente (Tabla 3). El grupo A está compuesto de 3 especies, siendo una de éstas constante en las estaciones del grupo (el bivalvo Austrochlamys natans), ninguna muy común, y dos comunes (el gastrópodo Trophon pallidus y el poliplacóforo Ischnochiton pusio), en tanto, no se presentaron especies exclusivas de este grupo (Tabla 3).

El grupo B está compuesto de 73 especies, de las cuales cinco son constantes (los gastrópodos Margarella violacea, Xymenopsis muriciformis y Pareuthria powelli, y los bivalvos Aulacomya atra y Zygochlamys patagonica) y ocho son muy comunes del grupo (los gastrópodos Nacella flammea,
Fissurella radiosa radiosa, Fissurella sp. juvenil, Fusitriton magellanicus, Meteuthria martensi, Pareuthria candidata y Pareuthria plumbea, y el bivalvo Austrochlamys natans; ver Tabla 3). Por su parte una gran cantidad de especies (27) fueron exclusivas de este grupo (ver Tabla 3 ).

El grupo C está constituido por 70 especies, de las cuales 16 son constantes (los gastrópodos Fissurella sp. juvenil, Margarella violacea, Eatoniella ebenina, Eatoniella sp., Xymenopsis muriciformis y Savatieria cf. meridionalis, los bivalvos Lissarca miliaris, Crenella magellanica, Mytilus edulis chilensis, Austrochlamys natans, Cyclochlamys multistriata, Neolepton concentricum, Neolepton yagan y Eurhomalea exalbida, y los poliplacóforos Leptochiton kerguelensis e Ischnochiton pusio) y 25 muy comunes (ver Tabla 3). Además, una gran cantidad de especies (25) fueron prácticamente exclusivas de las estaciones de este grupo (ver Tabla 3).

El grupo D está compuesto de 16 especies, una de estas constante y otra muy común (los gastrópodos Xymenopsis muriciformis y Margarella violacea, respectivamente). Por su parte, el bivalvo Cyamium sp. fue la única especie exclusiva del grupo (ver Tabla 3).

Solamente una especie (el gastrópodo Trophon pallidus) se presentó en todos los grupos (ver Tabla 3), no obstante, el bivalvo Aulacomya atra fue la especie con mayor presencia en los distintos sitios de muestreo (17 estaciones), seguida de los gastrópodos Margarella violacea, Xymenopsis muriciformis, Pareuthria powelli, y el bivalvo Zygochlamys patagonica (16 estaciones). En tanto, tres especies se presentaron exclusivamente en estaciones no agrupadas en ningún ensamble (estaciones CJ3s, CJ5s, CIII16s y PENBRU33s, ver Figs. 1, 4 y 5), los microgastrópodos Onoba cf. sulcula, Powellisetia microlirata y el bivalvo Brachidontes blakeanus (ver Tabla 3). 
Tabla 2. Resultados del análisis SIMPER, donde los grupos de estaciones fueron dispuestos de acuerdo a su similitud promedio (en negrita) y las especies de acuerdo a su contribución a la similitud dentro de cada grupo, para la acumulación de la similitud. Además, para cada especie fue calculada la abundancia promedio en cada grupo, la similitud promedio y la tasa del análisis (similitud/desviación estándar, DE). N.A., no aplica el índice.

\begin{tabular}{|c|c|c|c|c|c|}
\hline Especie & $\begin{array}{c}\text { Abundancia } \\
\text { promedio }\end{array}$ & $\begin{array}{l}\text { Similitud } \\
\text { promedio }\end{array}$ & Similitud / DE & Contribución (\%) & Acumulación (\%) \\
\hline Grupo C & & 54,00 & & & \\
\hline Neolepton yagan & 3,18 & 4,54 & 7,14 & 8,41 & 8,41 \\
\hline Eatoniella ebenina & 2,92 & 4,26 & 6,22 & 7,89 & 16,31 \\
\hline Xymenopsis muriciformis & 2,4 & 3,31 & 8,53 & 6,14 & 22,45 \\
\hline Eatoniella sp. & 2,21 & 2,93 & 8,97 & 5,43 & 27,88 \\
\hline Austrochlamys natans & 1,9 & 2,68 & 4,45 & 4,97 & 32,85 \\
\hline Neolepton concentricum & 2,36 & 2,67 & 2,26 & 4,94 & 37,79 \\
\hline Margarella violacea & 1,94 & 2,64 & 9,41 & 4,89 & 42,68 \\
\hline Lissarca miliaris & 1,92 & 2,36 & 4,68 & 4,36 & 47,04 \\
\hline Savatieria cf. meridionalis & 1,57 & 2,09 & 8,94 & 3,87 & 50,92 \\
\hline Leptochiton kerguelensis & 1,81 & 1,96 & 8,33 & 3,62 & 54,54 \\
\hline Fissurella sp. juvenil & 1,24 & 1,78 & 3,63 & 3,29 & 57,82 \\
\hline Eurhomalea exalbida & 1,27 & 1,74 & 11,8 & 3,22 & 61,04 \\
\hline Mytilus edulis chilensis & 1,2 & 1,69 & 7,03 & 3,13 & 64,17 \\
\hline Crenella magellanica & 1,38 & 1,59 & 8,94 & 2,94 & 67,11 \\
\hline Cyclochlamys multistriata & 1 & 1,59 & 8,94 & 2,94 & 70,05 \\
\hline Ischnochiton pusio & 1,19 & 1,59 & 8,94 & 2,94 & 72,99 \\
\hline Homalopoma cunninghami & 1,39 & 0,9 & 0,58 & 1,66 & 74,65 \\
\hline Philobrya cf. sublaevis & 1,13 & 0,84 & 0,58 & 1,55 & 76,2 \\
\hline Pareuthria candidata & 1,14 & 0,79 & 0,58 & 1,47 & 77,67 \\
\hline Meteuthria martensi & 0,94 & 0,69 & 0,58 & 1,28 & 78,95 \\
\hline Carditella naviformis & 1,32 & 0,69 & 0,58 & 1,28 & 80,23 \\
\hline
\end{tabular}

Grupo A

44,49

Austrochlamys natans

1,25

44,49

n.a.

100

100

\section{Grupo B}

38,49

Aulacomya atra

Zygochlamys patagonica

Margarella violacea

Xymenopsis muriciformis

Pareuthria powelli

3,45

3,45

1,26

1,21

1,32

1,24

0,88

Nacella flammea

0,93

Fusitriton magellanicus

Pareuthria plumbea

Fissurella radiosa radiosa

Austrochlamys natans

Meteuthria martensi

Mytilus edulis chilensis

Fissurella sp. juvenil

\section{Grupo D}

Xymenopsis muriciformis

Margarella violacea
0,95

0,84

0,86

1,04

0,72

0,85

0,68
3,17

2,92

2,66

2,66

2,56

2,19

2,04

1,95

1,42

1,19

1,14

\section{8,56}

1,37

15,01

\section{1,22}

1,01

1,04

1,08

1,06

0,92

0,88

0,94

0,73

0,71

0,73

0,47

0,57

$\begin{array}{cc}8,96 & 8,96 \\ 8,96 & 17,91 \\ 8,23 & 26,14 \\ 7,59 & 33,73 \\ 6,92 & 40,66 \\ 6,91 & 47,57 \\ 6,65 & 54,22 \\ 5,69 & 59,9 \\ 5,3 & 65,2 \\ 5,08 & 70,28 \\ 3,7 & 73,97 \\ 3,08 & 77,05 \\ 2,96 & 80,01\end{array}$

3,8

52,54

0,9

28,57

52,54 81,11 
TABLA 3. Índices de constancia $(\mathrm{C}, \%)$ y fidelidad $(\mathrm{F}, \%)$ en los grupos de estaciones. Especies raras $(\mathrm{C} \leq 12 \%)$, poco comunes $(C=13-25 \%)$, comunes $(C=26-50 \%)$, muy comunes $(C=51-75 \%)$ y constantes $(C=76-100 \%)$; especies accidentales $(F \leq$ $10 \%)$, ocasionales $(F=11-33 \%)$, accesorias $(F=34-50 \%)$, preferentes $(F=51-66 \%)$, electivas $(F=67-90 \%)$ y exclusivas $(F=$ 91-100\%); n.a., especies no agrupadas. Las especies constantes y exclusivas se destacan en negrilla.

\begin{tabular}{|c|c|c|c|c|}
\hline \multirow[t]{2}{*}{ Especie (Familia) } & Grupo A & Grupo B & Grupo C & Grupo D \\
\hline & $\mathrm{CF}$ & $\mathrm{CF}$ & $\mathrm{CF}$ & $\mathrm{CF}$ \\
\hline \multicolumn{5}{|l|}{ Gastropoda } \\
\hline Iothia emarginuloides (Lepetidae) & 00 & 3327 & 6753 & 2520 \\
\hline Nacella deaurata (Nacellidae) & 00 & 5100 & 00 & 00 \\
\hline Nacella flammea (Nacellidae) & 00 & 7144 & 6741 & 2515 \\
\hline Nacella mytilina (Nacellidae) & 00 & 19100 & 00 & 00 \\
\hline Nacella sp. juvenil (Nacellidae) & 00 & 2933 & 3338 & 2529 \\
\hline Scissurella clathrata (Scissurellidae) & 00 & 00 & 33100 & 00 \\
\hline Fissurella oriens oriens (Fissurellidae) & 00 & 2930 & 6770 & 00 \\
\hline Fissurella picta picta (Fissurellidae) & 00 & 1022 & 3378 & 00 \\
\hline Fissurella radiosa radiosa (Fissurellidae) & 00 & 62100 & 00 & 00 \\
\hline Fissurella sp. juvenil (Fissurellidae) & 00 & 5230 & 10056 & 2514 \\
\hline Puncturella conica (Fissurellidae) & 00 & 4339 & 6761 & 00 \\
\hline Calliostoma nudum (Calliostomatidae ) & 00 & 14100 & 00 & 00 \\
\hline Calliostoma sp. (Calliostomatidae ) & 00 & 5100 & 00 & 00 \\
\hline Margarella expansa (Calliostomatidae) & 00 & 10100 & 00 & 00 \\
\hline Margarella violacea (Calliostomatidae) & 00 & 7630 & 10040 & 7530 \\
\hline Submargarita cf. impervia (Turbinidae) & 00 & 00 & 33100 & 00 \\
\hline Tegula atra (Turbinidae) & 00 & 00 & 67100 & 00 \\
\hline Homalopoma cunninghami (Colloniidae) & 00 & 2930 & 6770 & 00 \\
\hline Laevilitorina caliginosa (Littorinidae) & 00 & 2421 & 6758 & 2522 \\
\hline Eatoniella denticula (Eatoniellidae) & 00 & 00 & 67100 & 00 \\
\hline Eatoniella ebenina (Eatoniellidae) & 00 & 1413 & $\mathbf{1 0 0 8 8}$ & 00 \\
\hline Eatoniella sp. (Eatoniellidae) & 00 & 1916 & 10084 & 00 \\
\hline Onoba scythei (Rissoidae) & 00 & 00 & 33100 & 00 \\
\hline Onoba cf. sulcula (Rissoidae) & 0n.a. & 0n.a. & 0n.a. & On.a. \\
\hline Powellisetia microlirata (Rissoidae) & 0n.a. & 0n.a. & On.a. & On.a. \\
\hline Caecum cf. chilensis (Caecidae) & 00 & 00 & 33100 & 00 \\
\hline Fartulum magellanicum (Caecidae) & 00 & 00 & 33100 & 00 \\
\hline Crepipatella dilatata [s.l.] (Calyptraeidae) & 00 & 2442 & 3358 & 00 \\
\hline Trochita pileolus (Calyptraeidae) & 00 & 14100 & 00 & 00 \\
\hline Euspira patagonica (Naticidae) & 00 & 57 & 6793 & 00 \\
\hline Tectonatica impervia (Naticidae) & 00 & 55 & 6769 & 2526 \\
\hline Argobuccinum ranelliforme (Ranellidae) & 00 & 24100 & 00 & 00 \\
\hline Fusitriton magellanicus (Ranellidae) & 00 & 7146 & 3322 & 5032 \\
\hline Eumetula pulla (Newtoniellidae) & 00 & 3350 & 3350 & 00 \\
\hline Trophon geversianus (Muricidae) & 00 & 24100 & 00 & 00 \\
\hline Trophon pallidus (Muricidae) & 5034 & 3826 & 3323 & 2517 \\
\hline Trophon plicatus (Muricidae) & 00 & 2933 & 3338 & 2529 \\
\hline Xymenopsis muriciformis (Muricidae) & 00 & 7628 & 10036 & 10036 \\
\hline Meteuthria martensi (Buccinidae) & 00 & 6248 & 6752 & 00 \\
\hline Pareuthria candidata (Buccinidae) & 00 & 5244 & 6756 & 00 \\
\hline Pareuthria cerealis (Buccinidae) & 00 & 3333 & 6767 & 00 \\
\hline Pareuthria michaelseni (Buccinidae) & 00 & 14100 & 00 & 00 \\
\hline Pareuthria paessleri (Buccinidae) & 00 & 5100 & 00 & 00 \\
\hline Pareuthria plumbea (Buccinidae) & 00 & 7152 & 6748 & 00 \\
\hline Pareuthria powelli (Buccinidae) & 00 & 7653 & 6747 & 00 \\
\hline
\end{tabular}




\begin{tabular}{|c|c|c|c|c|}
\hline \multirow[t]{2}{*}{ Especie (Familia) } & Grupo A & Grupo B & Grupo C & Grupo D \\
\hline & CF & CF & CF & CF \\
\hline Savatieria cf. meridionalis (Buccinidae) & 00 & 00 & 10080 & 2520 \\
\hline Nassarius cf. coppingeri (Nassariidae) & 00 & 5100 & 00 & 00 \\
\hline Adelomelon ancilla (Volutidae) & 00 & 10100 & 00 & 00 \\
\hline Admete schythei (Cancellariidae) & 00 & 5100 & 00 & 00 \\
\hline Belalora cunninghami (Mangeliidae) & 00 & 00 & 33100 & 00 \\
\hline Typhlodaphne payeni (Borsoniidae) & 00 & 00 & 67100 & 00 \\
\hline Typhlodaphne strebeli (Borsoniidae) & 00 & 1430 & 3370 & 00 \\
\hline Mathilda magellanica (Mathildidae) & 00 & 00 & 67100 & 00 \\
\hline Odostomia sp. (Pyramidellidae) & 00 & 00 & 33100 & 00 \\
\hline Turbonilla strebeli (Pyramidellidae) & 00 & 00 & 33100 & 00 \\
\hline Diaphana paessleri (Diaphanidae) & 00 & 513 & 3388 & 00 \\
\hline Toledonia perplexa (Diaphanidae) & 00 & 00 & 67100 & 00 \\
\hline Kerguelenella lateralis (Siphonariidae) & 00 & 14100 & 00 & 00 \\
\hline Siphonaria lessoni (Siphonariidae) & 00 & 5100 & 00 & 00 \\
\hline \multicolumn{5}{|l|}{ Bivalvia } \\
\hline Nucula falklandica (Nuculidae) & 00 & 00 & 33100 & 00 \\
\hline Neilonella sulculata (Neilonellidae) & 00 & 14100 & 00 & 00 \\
\hline Lissarca miliaris (Philobryidae) & 00 & 00 & 100100 & 00 \\
\hline Philobrya cf. sublaevis (Philobryidae) & 00 & 3836 & 6764 & 00 \\
\hline Aulacomya atra (Mytilidae) & 00 & 81100 & 00 & 00 \\
\hline Brachidontes blakeanus (Mytilidae) & 0n.a. & On.a. & 0n.a. & On.a. \\
\hline Crenella magellanica (Mytilidae) & 00 & 00 & 100100 & 00 \\
\hline Mytilus edulis chilensis (Mytilidae) & 00 & 4832 & 10068 & 00 \\
\hline Brachidontes purpuratus (Mytilidae) & 00 & 14100 & 00 & 00 \\
\hline Limatula deceptionensis (Limidae) & 00 & 2426 & 6774 & 00 \\
\hline Austrochlamys natans (Pectinidae) & 10038 & 6224 & 10038 & 00 \\
\hline Zygochlamys patagonica (Pectinidae) & 00 & 7648 & 3321 & 5031 \\
\hline Cyclochlamys multistriata (Propeamussiidae) & 00 & 00 & 100100 & 00 \\
\hline Epicodakia falklandica (Lucinidae) & 00 & 00 & 33100 & 00 \\
\hline Lasaea adansoni (Lasaeidae) & 00 & 1430 & 3370 & 00 \\
\hline Mysella sp. (Montacutidae) & 00 & 00 & 33100 & 00 \\
\hline Neolepton amatoi (Neoleptonidae) & 00 & 00 & 33100 & 00 \\
\hline Neolepton concentricum (Neoleptonidae) & 00 & 109 & 10091 & 00 \\
\hline Neolepton yagan (Neoleptonidae) & 00 & 1413 & 10088 & 00 \\
\hline Neolepton sp. (Neoleptonidae) & 00 & 24100 & 00 & 00 \\
\hline Cyamiocardium dahli (Cyamiidae) & 00 & 00 & 67100 & 00 \\
\hline Cyamium sp. (Cyamiidae) & 00 & 00 & 00 & 25100 \\
\hline Gaimardia trapesina (Cyamiidae) & 00 & 00 & 67100 & 00 \\
\hline Carditella naviformis (Carditidae) & 00 & 2930 & 6770 & 00 \\
\hline Carditopsis flabellum (Condylocardiidae) & 00 & 1013 & 6788 & 00 \\
\hline Astarte longirostra (Astartidae) & 00 & 5100 & 00 & 00 \\
\hline Ameghinomya antiqua (Veneridae) & 00 & 10100 & 00 & 00 \\
\hline Clausinella gayi (Veneridae) & 00 & 1022 & 3378 & 00 \\
\hline Eurhomalea exalbida (Veneridae) & 00 & 107 & 10074 & 2519 \\
\hline Pitar rostratus (Veneridae) & 00 & 00 & 33100 & 00 \\
\hline Hiatella antarctica (Lyonsiidae) & 00 & 14100 & 00 & 00 \\
\hline \multicolumn{5}{|l|}{ Polyplacophora } \\
\hline Leptochiton kerguelensis (Leptochitonidae) & 00 & 1913 & 10069 & 2517 \\
\hline Ischnochiton pusio (Ischnochitonidae) & 5030 & 1911 & $\mathbf{1 0 0 5 9}$ & 00 \\
\hline Ischnochiton punctulatissimus (Ischnochitonidae) & 00 & 1013 & 6788 & 00 \\
\hline
\end{tabular}




\begin{tabular}{lcccc}
\hline Especie (Familia) & Grupo A & Grupo B & Grupo C & Grupo D \\
\cline { 2 - 5 } & CF & CF & CF & CF \\
\hline Ischnochiton stramineus (Ischnochitonidae) & 00 & 1022 & 3378 & 00 \\
Callochiton puniceus (Callochitonidae) & 00 & $14 \mathbf{1 0 0}$ & 00 & 00 \\
Chiton bowenii (Chitonidae) & 00 & $5 \mathbf{1 0 0}$ & 00 & 00 \\
Tonicia lebruni (Chitonidae) & 00 & 513 & 3388 & 00 \\
Tonicia smithi (Chitonidae) & 00 & $5 \mathbf{1 0 0}$ & 00 & 00 \\
Tonicia sp. juvenil (Chitonidae) & 00 & 1022 & 3378 & 00 \\
Nuttalochiton martiali (Mopaliidae) & 00 & $14 \mathbf{1 0 0}$ & 00 & 00 \\
Plaxiphora aurata (Mopaliidae) & 00 & 1022 & 3378 & 00 \\
\hline
\end{tabular}

\section{DISCUSIÓN}

Número y composición de especies

Valdovinos (1999) en su base de datos taxonómica y distribucional citó un total de 959 especies marinas (671 gastrópodos, 226 bivalvos y 63 poliplacóforos) para el territorio nacional de Chile, incluyendo especies antárticas y de islas oceánicas; de este total, 437 especies (302 gastrópodos, 99 bivalvos y 36 poliplacóforos) las registró para la franja latitudinal 50-55ㅇ. Las 101 especies encontradas en este estudio (59 gastrópodos y 31 bivalvos y 11 poliplacóforos) corresponden al $\sim 11 \%$ de las especies citadas para Chile ( $9 \%$ de los gastrópodos totales, $\sim 14 \%$ de bivalvos totales y $\sim 17 \%$ de poliplacóforos totales). Regionalmente, corresponden a un $~ 23 \%$ de las especies citadas por Valdovinos (1999) para la franja latitudinal 50-55오 ( 20\% de los gastrópodos, $\sim 31 \%$ de bivalvos y $\sim 31 \%$ de poliplacóforos), y a un $24 \%$ de las especies de gastrópodos y bivalvos citadas por Linse (1999) para la región Magallánica, definida como la plataforma patagónica al sur de $41^{\circ}$ ㅇ en los márgenes Pácifico y Atlántico de Sudamérica. Sin embargo, dichos porcentajes pueden ser sólo considerados como referencia, puesto que ambos reportes (Linse 1999, Valdovinos 1999) incluyen desde especies intermareales a abisales y algunas especies que actualmente pueden ser consideradas sinónimos júnior de otras, luego de revisiones taxonómicas de grupos específicos. Por tanto, la cantidad de especies ha variado, más bien tendiendo a decrecer en algunos casos (ver por ejemplo, Zelaya \& Ituarte 2004, Pastorino 2005a, 2005b).

La cantidad de especies de moluscos registrados en este trabajo representa alrededor de cuatro veces el promedio de aquellas reportadas en otros trabajos realizados en los últimos 40 años en ambientes sublitorales en el Estrecho de Magallanes y canales adyacentes (Tabla 4). Al respecto, el estudio que más se aproxima en la cantidad de especies es el de Ríos et al. (2003), quienes en un área muy acotada de la micro-cuenca oriental del estrecho registraron 69 especies entre 30 y 50 metros (ver Tabla 4). Dell (1971) reportó 73 especies en una extensiva zona de fiordos y canales entre $42^{\circ} \mathrm{S}$ y 55으, pero no consideró los fondos del Estrecho de Magallanes. Otros trabajos reportan moluscos para la zona, pero lo hacen desde un punto de vista de la abundancia y biomasa sin entregar número de especies (e.g. Montiel et al. 2001).

La familia con mayor número de especies reportadas (i.e. Buccinidae) responde a la gran diversidad que presentan en la Región Magallánica.

Tabla 4. Moluscos registrados en trabajos desde 1970 en el estrecho de Magallanes y canales adyacentes, tomando en cuenta estudios donde fueron recolectadas muestras sublitorales.

\begin{tabular}{|c|c|c|c|c|}
\hline \multirow[t]{2}{*}{ Publicación } & \multirow[t]{2}{*}{ Latitud y profundidad } & \multicolumn{3}{|c|}{ Número de especies } \\
\hline & & Gastropoda & Bivalvia & Polyplacophora \\
\hline Linse (2002) & 52,9-53,7오; 8-522m & 17 & 1 & No reportado \\
\hline Ríos et al. (2003) & $52,6-52,8^{\circ} \mathrm{S} ; 30-50 \mathrm{~m}$ & 38 & 21 & 10 \\
\hline Ríos et al. (2005) & $52,3-53,9^{\circ} \mathrm{S} ; 2^{24-604 m}$ & 8 & 6 & 1 \\
\hline Ríos et al. (2007) & $53,0-53,6^{\circ} \mathrm{S} ; \sim 8 \mathrm{~m}$ & 9 & 5 & 4 \\
\hline Thatje \& Brown (2009) & $52,3-55,2^{\circ}$ S; 35-571m & 5 & 15 & 1 \\
\hline Ríos et al. (2010) & $52,3-52,5^{\circ} \mathrm{S} ; \sim 16-\sim 61 \mathrm{~m}$ & 1 & 3 & 0 \\
\hline Este estudio & 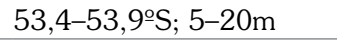 & 59 & 31 & 11 \\
\hline
\end{tabular}


Linse (1999) reportó la presencia de 27 especies de Buccinidae (como Buccinulidae) para la región de Magallanes. Por otro lado, la especie más abundante obtenida (Margarella violacea) se ha reportado como especie de considerable abundancia en trabajos anteriores (e.g. Ríos et al. 2003).

\section{Diversidad y agrupamientos}

Los valores obtenidos para los índices de diversidad han resultado ser altos en comparación con información previa obtenida para otros sectores sublitorales del estrecho de Magallanes (e.g. Gutt et al. 1999), pero comparables al estudio de Ríos et al. (2003) de fondos sublitorales en la cuenca oriental, utilizando todos los invertebrados bentónicos. Adicionalmente, se ha logrado agregar en la última década un mayor esfuerzo de muestreo, mayoritariamente de tipo cuantitativo (e.g. Montiel et al. 2001, Thatje \& Mutschke 1999a, Montiel et al. 2011).

La diversidad de especies podría directamente responder a la heterogeneidad de hábitats presentes en la región de Magallanes. Se ha señalado que bajo condiciones constantes se espera que la diversidad específica aumente en zonas con heterogeneidad del hábitat (Gray 2001). La particular geomorfología de la zona con sus distintos sustratos, paredes, depresiones, morrenas y aporte de agua dulce desde glaciares, crea condiciones ambientales locales particulares que pueden originar claras diferencias estructurales en las comunidades faunísticas, incluso a cortas distancias (Benedetti-Cecchi \& Cinelli 1997, Gutt et al. 1999, Gutt et al. 2003).

Considerando la conformación de los ensambles obtenidos, no se encontró un patrón de estructuración u ordenamiento que pueda ser relacionado a ciertas características ambientales (no consideradas en el estudio), sino que más bien se estructuró un gran ensamble (grupo B, ver Figs. 4 y 5) formado por la mayoría de las estaciones del área de estudio. Al respecto, tomando en cuenta la geomorfología costera de las zonas muestreadas, destacan una gran cantidad de fiordos y canales pequeños, que dan una gran heterogeneidad al lugar, pero no indicarían un patrón de distribución. En este sentido, otros podrían ser los factores relacionados a la estructuración de las comunidades. Varios autores han señalado que los ensambles bentónicos están controlados por tres factores principales: suministro alimenticio, temperatura y régimen sedimentario (e.g. Brey \& Clarke 1993, Sáiz- Salinas et al. 1997). Factores locales como la temperatura y el alimento y sus oscilaciones estacionales influyen en el metabolismo y crecimiento de los invertebrados marinos y entonces son importantes factores que estructuran los ensambles bentónicos (Brey \& Clarke 1993). El régimen sedimentario está relacionado a la presencia de glaciares en las zona de fiordos (Ríos et al. 2005), por efecto de cambios en los regimenes hidrográficos y procesos de sedimentación producidos por derretimiento de glaciares (Montiel et al. 2005), observándose comunidades más empobrecidas en fiordos y más ricas en canales (Thatje \& Mutschke 1999b).

\section{Aspectos taxonómicos}

El gran número de especies obtenidas también se ve reflejado por los avances en investigaciones taxonómicas realizadas en el último tiempo en la región, que permiten identificar especies con precisión. Por ejemplo, muchos de los grupos identificados han sido revisados: Protobranchia (Villarroel \& Stuardo 1998), Naticidae (Pastorino 2005a), Neoleptonidae (Zelaya \& Ituarte 2003, 2004), Scissurellidae (Zelaya \& Geiger 2007), Trophon (Pastorino 2005b), Xymenopsis (Pastorino \& Harasewych 2000). Contrariamente, algunas identidades no se pudieron identificar a nivel específico, principalmente por tratarse de ejemplares en estados de desarrollo intermedio (e.g. Fissurella sp. juvenil, Nacella sp. juvenil, ver Tabla 3) o por constituir grupos que no han sido revisados taxonómicamente (e.g. Savatieria cf. meridionalis, Odostomia sp., ver Tabla 3).

\section{CONCLUSIONES}

Los resultados obtenidos en el presente estudio y su consiguiente comparación con trabajos anteriores permiten concluir que la diversidad de moluscos bentónicos registrados es notoriamente más alta que la reportada en otros sectores del Estrecho de Magallanes. No obstante la organización de los ensambles obtenidos no permite definir un patrón en el área de estudio, sugiriendo considerar parámetros abióticos en futuras investigaciones y una mayor cantidad de muestreos en áreas adyacentes. 


\section{AGRADECIMIENTOS}

Los autores desean expresar su gratitud a la Fundación CEQUA, entidad ejecutora del proyecto "Diagnóstico del Macrobentos en el Área Marina Costera Protegida de Múltiples Usos Francisco Coloane" (Código BIP N 30061434-0), soporte de la información para la realización de este trabajo; a la Secretaría Regional Ministerial de Bienes Nacionales de la Región de Magallanes, quienes financiaron el proyecto; a todos los colegas que participaron en las distintas etapas de la investigación; al investigador Carlos Olave (Fundación CEQUA), por la proporción de mapas e información geográfica y al Proyecto FONDECYT 3120082.

\section{LITERATURA CITADA}

Aldea, C. \& S. Rosenfeld 2011. Moluscos intermareales de la Playa Buque Quemado (Estrecho de Magallanes, Chile). Revista de Biología Marina y Oceanografía, 46(2): 115-124

Aldea, C., T. Césped \& S. Rosenfeld 2011. Opisthobranchs from Bernardo O'Higgins National Park (S. Chile). Thalassas, 27(2): 37-48

Antezana, T. 1999. Hydrographic features of Magellan and Fuegian island passages and adjacent Subantarctic waters. Scientia Marina, 63(Suppl.1): 23-34

Appeltans, W., P. Bouchet, G.A. Boxshall, K. Fauchald, D.P. Gordon, B.W. Hoeksema, G.C. B. Poore, R.W.M. Van Soest, S. Stöhr, T.C. Walter \& M. J. Costello (eds.) 2011. World Register of Marine Species. http://www. marinespecies.org. Revisada el 01-10-2011.

Arnaud, P. M., J. S. Troncoso \& A. Ramos 2001. Species diversity and assemblages of macrobenthic Mollusca from the South Shetland Islands and Bransfield Strait (Antarctica). Polar Biology, 24:105-112

Arntz, W. \& M. Gorny 1996. Cruise Report of the Joint Chilean-German-Italian Magellan "Victor Hensen" Campaign in 1994. Beritchte zur Polarforschung,190:1-113

Benedetti-Cecchi, L. \& F. Cinelli 1997. Spatial distribution of algae and invertebrates in the rocky intertidal zone of the Strait of Magellan: are patterns general? Polar Biology, 18: $337-343$
Bray, R.J. \& J.I. Curtis 1957. An ordination of the upland forest communities of southern Wisconsin. Ecological Monographs, 27: 325-349

Brey, T. \& A. Clarke 1993. Population dynamics of marine benthic invertebrates in Antarctic and subantarctic environments: are there unique adaptations? Antarctic Science, 5(3):253-266

Camus, P. A. 2001. Biogeografía marina de Chile. Revista Chilena de Historia Natural, 74: 587-617

Cárdenas, C. 2008. Factores que organizan la estructura comunitaria del megaepibentos del submareal rocoso de Punta Santa Ana, Estrecho de Magallanes, Chile. Tesis de Magíster en Ciencias con mención en Manejo y Conservación de Recursos Naturales de Ambientes Subantárticos. Universidad de Magallanes, Chile. 109 pp.

Chen, G.T., R.L. Herman \& M. Vincx 1999. Meiofauna communities from the Straits of Magellan and the Beagle Channel. Scientia Marina, 63 (1): 123-132-

Clarke, K.R. 1993. Non-parametric multivariate analyses of changes in community structure. Australian Journal of Ecology, 18: 117-143

Clarke, K.R. \& R.H. Green 1988. Statistical design and analysis for a "biological effects" study. Marine Ecology Progress Series, 46: 213-226

Clarke, K.R. \& R.N. Gorley 2005. Primer-E version 6.0. Plymouth, UK: Natural Environmental Research Council, Plymouth Marine Laboratory. $91 \mathrm{pp}$.

Clarke, K. R., P.J. Somerfield \& R.N. Gorley 2008. Testing of null hypotheses in exploratory community analyses: similarity profiles and biota-environment linkage. Journal of Experimental Marine Biology and Ecology, 366: 56-69

Dajoz, R. 1971. Précis d'écologie. 2o éd. Dunod, Paris. 434 pp.

Dell, R. K. 1971. The marine Mollusca of the Royal Society Expedition to southern Chile, 19581959. Records of the Dominion Museum, 7(17): 155-233

Fernández, M., E. Jaramillo, P.A. Marquet, C.A. Moreno, S.A. Navarrete, F.P. Ojeda, C. Valdovinos \& J.A. Vásquez 2000. Diversity, dynamics and biogeography of Chilean ben- 
thic nearshore ecosystems: an overview and guidelines for conservation. Revista Chilena de Historia Natural, 73: 629-662

Field, J. G., K. R. Clarke, \& R. M. Warwick 1982. A practical strategy for analysing multispecies distributions patterns. Marine Ecology Progress Series, 8: 37-52

Gerdes, D. \& A. Montiel 1999. Distribution patterns of macrozoobenthos: a comparison between the Magellan region and Weddell Sea (Antarctica). Scientia Marina, 63(1): 149-154

Gray, J. S. 1997. Marine biodiversity: patterns, threats and conservation needs. Biodiversity and Conservation, 6: 153-175

Gray, J. S. 2001. Marine diversity: the paradigms in patterns of species richness examined. Scientia Marina, 65: 41-56

Gutt, J., E. Helsen, W. Arntz \& A. Buschmann 1999. Biodiversity and community structure of the mega-epibenthos in the Magellan region (South America). Scientia Marina, 63 (Suppl. 1): 155-170

Gutt, J., W. Arntz, E. Balguerias, A. Brandt, D. Gerdes, M. Gorny \& B. Sirenko 2003. Diverse approaches to questions of biodiversity: German contributions to studies of marine benthos around South America and Antarctica. Gayana, 67(2): 177-189

Hromic, T. 2009. Distribución batimétrica de foraminíferos bentónicos (Protozoa: Foraminiferida) al Sur del Estrecho de Magallanes $\left(52^{\circ}-56^{\circ} \mathrm{S}\right)$, Chile. Anales Instituto Patagonia (Chile) 37(1): 23-38

King, P.P. \& W.J. Broderip 1832. Description of the Cirripedia, Conchifera and Mollusca in a collection formed by the officies of HMS Adventure and Beagle employed between the years 1826 and 1830 in surveying the southern coast of South America including the Strait of Magellan and the coast of Tierra del Fuego. Zoological Journal, 5:332-349

Kruskal, J. B. \& M. Wish 1978. Multidimensional scaling. Sage Publications Inc. Beverly Hills, California.

Lancellotti, D. A. \& J. A. Vásquez 2000 Zoogeografía de macroinvertebrados bentónicos de la costa de Chile: contribución para la conservación marina. Revista Chilena de Historia Natural, 73:99-129
Linse, K. 1997. Die Verbreitung epibenthischer Mollusken im chilennischen Beagle -Kanal. Berichte zur Polarforschung, 228:1-131

Linse, K. 1999. Mollusca of the Magellan region. A cheklist of the species and their distribution. Scientia Marina, 63 (Supl.1): 399-407

Linse, K. 2002. The shelled Magellanic Mollusca: with special reference to biogeography relations in the Southern Ocean, Theses Zoologicae, 34:1-252. A.R.G. Gantner Verlag KG, Ruggell, Lichtenstein.

Lorenti, M. \& S. Mariani 1997. Isopod assemblages in the Straits of Magellan: structural and functional aspects. Polar Biology, 18:254-259

Mazzocchi, M. G. \& A. Ianora 1991. A faunistic study of the copepod assemblages in the Strait of Magellan. Bolletino Di Oceanologia Teorica ed Applicata, 9: 163-177

Montiel, A., D. Gerdes \& C. Ríos 2001. Distribución y abundancia del macrozoobentos en una microcuenca marina submareal del Estrecho de Magallanes, Chile. Anales Instituto de la Patagonia (Chile), 29:117-133

Montiel, A., D. Gerdes, B. Hilbig \& W.E. Arntz 2005. Polychaete assemblages on the Magellan and Weddell Sea shelves: comparative ecological evaluation. Marine Ecology Progress Series, 297:189-202

Montiel, A., C. Ríos \& E. Mutschke 2007. Biodiversidad de los poliquetos sublitorales de las costas noroccidental y sur de la isla de Tierra del Fuego (Chile). Anales Instituto de la Patagonia (Chile) 35:41-52

Montiel, A., E. Quiroga \& D. Gerdes 2011. Diversity and spatial distribution patterns of polychaete assemblages in the Paso Ancho, Straits of Magellan Chile. Continental Shelf Research, 31: 304-314

Mutschke, E. \& C. Ríos 2006. Distribución espacial y abundancia relativa de equinodermos en el estrecho de Magallanes, Chile. Ciencia y Tecnología del Mar, 29(1): 91-102

Mutschke, E., C. Ríos, \& A. Montiel 1998. Situación actual de la macrofauna presente en el intermareal de bloques y cantos de Bahía Laredo, Estrecho de Magallanes. Anales Instituto Patagonia Serie Cs. Nat. (Chile), 26: 5-29 
Pastorino, G. 2005a. Recent Naticidae (Mollusca: Gastropoda) from the Patagonian Coast. The Veliger, 47(4): 225-258

Pastorino, G. 2005b. A revision of the genus Trophon Monfort, 1810 (Gastropoda: Muricidae) from southern South America. The Nautilus, 119(2):55-82

Pastorino, G. \& M. G. Harasewych 2000. A revision of the Patagonian genus Xymenopsis Powell 1951. The Nautilus, 114(2):38-58

Pielou, E. C. 1966. The measurement of diversity in different types of biological collections. Journal of Theoretical Biology, 13: 131-144

Purvis, A. \& A. Hector 2000. Getting the measure of biodiversity. Nature, 405: 212-219

Reid, D. \& C. Osorio 2000. The shallow-water marine Mollusca of the Estero Elefantes and Laguna San Rafael southern Chile. Bulletin of the Natural History Museum of London (Zoology) 66(2): 109-146

Ríos, C. \& D. Gerdes 1997. Ensamble bentónico epifaunístico de un campo intermareal de bloques y cantos en Bahía Laredo, Estrecho de Magallanes. Anales del Instituto de la Patagonia, Serie Ciencias Naturales, 25: 47-55

Ríos, C. \& E. Mutschke 1999. Community structure of intertidal boulder-cobble fields in the Straits of Magellan, Chile. Scientia Marina, 63 (1):193-201

Ríos, C., E. Mutschke \& E. Morrison 2003. Biodiversidad bentónica sublitoral en el estrecho de Magallanes, Chile. Revista de Biología Marina y Oceanografía, 38 (1): 1-12

Ríos, C., E. Mutschke, A. Montiel, D. Gerdes \& W.E. Arntz 2005. Soft-bottom macrobenthic faunal associations in the southern Chilean glacial fjord complex. Scientia Marina, 69 (2):225-236

Ríos, C., W. E. Arntz, D. Gerdes, E. Mutschke \& A. Montiel 2007. Spatial and temporal variability of the benthic assemblages associated to the holdfasts of the kelp Macrocystis pyrifera in the Straits of Magellan, Chile. Polar Biology, 31(1):89-100

Ríos, C., E. Mutschke \& A. Montiel 2010. Estructura de la comunidad macrofaunística bentónica en la boca oriental del estrecho de Magallanes, Chile austral. Anales Instituto Patagonia (Chile) 38(1):83-96
Rohlf, F.J. 1963. Classification of Aedes by numerical taxonomic methods (Diptera: Culicidae). Annals of the Entomological Society of America, 56: 798-804

Sáiz-Salinas, J. I., A. Ramos, F. J. García, J. S. Troncoso, G. San Martin \& C. Palacin 1997. Quantitative analyses of macrobenthic softbottom assemblages in South Shetland waters (Antarctica). Polar Biology, 17: 393-400

Shannon, C. E. 1948. A mathematical theory of communication. Bell System Technical Journal, 27: 379-423

Thatje, S. \& E. Mutschke 1999a. Macrofauna bentónica muestreada con un Reineck box corer. Resultados Crucero Cimar-Fiordo 3, Comité Oceanográfico Nacional, Valparaíso, Chile: $126-128$

Thatje, S. \& E. Mutschke 1999b. Distribution of abundance, biomass, production and productivity of macrobenthos in the Subantarctic Magellan Province (South America). Polar Biology, 22: 31-37

Thatje, S. \& A. Brown 2009. The macrobenthic ecology of the Straits of Magellan and the Beagle Channel. Anales Instituto Patagonia (Chile) 37(2):17-27

Valdenegro, A. \& N. Silva 2003. Caracterización oceanográfica física y química de la zona de canales y fiordos australes de Chile entre el Estrecho de Magallanes y Cabo de Hornos (CIMAR 3 Fiordos). Ciencia y Tecnología del Mar, 26(2): 19-60

Valdovinos, C. 1999. Biodiversidad de moluscos chilenos: Base de datos taxonómica y distribución. Gayana, 63(2): 111-164

Villarroel, M. \& J. Stuardo 1998. Protobranchia (Mollusca: Bivalvia) chilenos recientes y algunos fósiles. Malacologia, 40(1-2): 113-229

Warwick, R. M. \& K. R. Clarke 1995. New 'biodiversity' measures reveal a decrease in taxonomic distinctness with increasing stress. Marine Ecology Progress Series, 129: 301-305

Zaixso, H., R. León, M. Palacios, C. Cárdenas, C. Olave, T. Hromic, J. Cárdenas, C. Aldea, J. Araos, M. Hüne, C. Valdovinos, A. Mansilla, N. Navarro, F. Valdivia, I. Cañete, S. Oyarzún, J. Plana, M. Santana, M. Gallardo, H. Duamante, S. Rosenfeld, M. Witto \& H. Hidalgo 2007. Diagnóstico del Macrobentos en el 
Área Marina Costera Protegida de Múltiples Usos Francisco Coloane, Código BIP $N^{\circ}$ 30061434-0; Informe Final. Fundación CEQUA, Centro de Estudios del Cuaternario Fuego Patagonia y Antártica, Punta Arenas, Chile, $218 \mathrm{pp}$.

Zelaya, D. 2004. The genus Margarella Thiele, 1893 (Gastropoda: Trochidae) in the southwestern Atlantic ocean. The Nautilus, 118(3): 112-120

Zelaya, D. 2010. New species of Thyasira, Mendicula, and Axinulus (Bivalvia, Thyasiroidea) from Sub-Antarctic and Antarctic waters. Polar Biology, 33: 607-616
Zelaya, D. \& C. Ituarte 2003. Two new species of Neolepton Monterosato, 1875 (Bivalvia: Neoleptonidae) from South Georgia Islands, South atlantic Ocean. The Nautilus, 117(1): 6-11

Zelaya, D. \& C. Ituarte 2004. The genus Neolepton Monterosato, 1875 in southern South America (Bivalvia: Neoleptonidae). Journal of Molluscan Studies, 70: 123-137

Zelaya, D. \& D. L. Geiger 2007. Species of Scissurellidae and Anatomidae from Sub-Antarctic and Antarctic waters (Gastropoda: Vetigastropoda). Malacologia, 49(2): 393-443 
